\title{
STAT2 signaling as double-edged sword restricting viral dissemination but driving severe pneumonia in SARS-CoV-2 infected hamsters
}

Robbert Boudewijns ${ }^{1,2 \#}$, Hendrik Jan Thibaut ${ }^{1,3 \#, *}$, Suzanne J. F. Kaptein ${ }^{1 \#}$, Rong Li ${ }^{4 \#}$, Valentijn Vergote $^{1,5,6}$, Laura Seldeslachts ${ }^{9}$, Carolien De Keyzer ${ }^{1,2}$, Sapna Sharma ${ }^{1,2}$, Sander Jansen ${ }^{1,2}$, Johan Van Weyenbergh $^{5}$, Ji Ma ${ }^{1,2}$, Erik Martens ${ }^{13}$, Lindsey Bervoets ${ }^{1}$, Tina Van Buyten ${ }^{1}$, Sofie Jacobs ${ }^{1}$, Yanan Liu $^{4}$, Joan Martí-Carreras ${ }^{5,6}$, Bert Vanmechelen ${ }^{5,6}$, Tony Wawina-Bokalanga ${ }^{5,6}$, Leen Delang ${ }^{1}$, Joana Rocha-Pereira ${ }^{1}$, Lotte Coelmont ${ }^{1,2}$, Winston Chiu ${ }^{1}$, Pieter Leyssen ${ }^{1}$, Elisabeth Heylen ${ }^{1}$, Dominique Schols ${ }^{1}$, Lanjiao Wang ${ }^{5,7}$, Lila Close ${ }^{5,7}$, Jelle Matthijnssens ${ }^{5,7}$, Marc Van Ranst ${ }^{5,14,15,16}$, Georg Schramm ${ }^{10,11}$, Koen Van Laere ${ }^{10,11}$, Ghislain Opdenakker ${ }^{13}$, Piet Maes ${ }^{5,6}$, Birgit Weynand ${ }^{8,12}$, Christopher Cawthorne ${ }^{10}$, Greetje Vande Velde ${ }^{9}$, Zhongde Wang ${ }^{4, \&}$, Johan Neyts ${ }^{1,2, \&, *}$, Kai Dallmeier ${ }^{1,2, *}$

${ }^{1}$ KU Leuven Department of Microbiology, Immunology and Transplantation, Rega Institute, Laboratory of Virology and Chemotherapy, B-3000 Leuven, Belgium

${ }^{2}$ Molecular Vaccinology and Vaccine Discovery Group

${ }^{3}$ Translational Platform Virology and Chemotherapy

${ }^{4}$ Department of Animal, Dairy, and Veterinary Sciences, Utah State University, UT 84322-4815

Logan, Utah, United States of America

${ }^{5}$ KU Leuven Department of Microbiology, Immunology and Transplantation, Rega Institute, Laboratory of Clinical and Epidemiological Virology, B-3000 Leuven, Belgium

${ }^{6}$ Zoonotic Infectious Diseases Unit

${ }^{7}$ Laboratory of Viral Metagenomics

${ }^{8}$ KU Leuven Department of Imaging and Pathology, Translational Cell and Tissue Research, B-3000 Leuven, Belgium

${ }^{9}$ KU Leuven Department of Imaging and Pathology, Biomedical MRI and MoSAIC, B-3000 Leuven, Belgium

${ }^{10}$ KU Leuven Department of Imaging and Pathology, Nuclear Medicine and Molecular Imaging and MoSAIC, B-3000 Leuven, Belgium

${ }^{11}$ Division of Nuclear Medicine, University Hospitals Leuven, B-3000 Leuven, Belgium

${ }^{12}$ Division of Translational Cell and Tissue Research

${ }^{13}$ KU Leuven Department of Microbiology, Immunology and Transplantation, Rega Institute, Immunity and Inflammation Research Group, Immunobiology Unit, B-3000 Leuven, Belgium

${ }^{14}$ KU Leuven Department of Laboratory Medicine, University Hospitals Leuven, B-3000 Leuven, Belgium

${ }^{15}$ National Reference Center for Respiratory Pathogens and Enteroviruses, B-3000 Leuven, Belgium

${ }^{16}$ Leuven University Vaccinology Center (LUVAC), 3000 Leuven, Belgium

${ }^{17}$ KU Leuven Department of Clinical and Experimental Medicine, Division of Respiratory Diseases, University Hospitals Leuven, B-3000 Leuven, Belgium

\footnotetext{
\# Equal contribution (co-first authors)

${ }^{\&}$ Co-senior authors
} 
*Correspondence: kai.dallmeier@kuleuven.be; hendrikjan.thibaut@kuleuven.be; johan.neyts@kuleuven.be 


\section{Introductory paragraph}

Since the emergence of SARS-CoV-2 causing COVID-19, the world is being shaken to its core with numerous hospitalizations and prospected hundreds of thousands of deaths. In search for key targets of effective therapeutics, robust animal models mimicking COVID-19 in humans are urgently needed. Here, we show that productive SARS-CoV-2 infection in the lungs of mice is limited and restricted by early type I interferon responses. In contrast, we show that Syrian hamsters are highly permissive to SARS-CoV-2. In wild-type hamsters, SARS-CoV-2 infection triggers bronchopneumonia and a strong inflammatory response in the lungs with neutrophil infiltration and edema. We further assess SARS-CoV-2-induced lung pathology in hamsters by micro-CT alike used in clinical practice. Finally, we identify an exuberant innate response as key player in immune pathogenesis, in which STAT2 signaling plays a double-edged role, driving severe lung injury on the one hand, yet restricting systemic virus dissemination on the other. Our results endorse hamsters as pre-clinical model to rationalize and assess the therapeutic benefit of new antivirals or immune modulators for the treatment of COVID-19 patients.

\section{Keywords}

SARS-CoV-2 (2019-nCoV), COVID-19, animal models, pneumonia, innate immunity, STAT2, IL28R, type I and III interferons, immune pathogenesis, micro-CT

\section{Introduction and Results}

SARS-CoV-2 (formerly 2019-nCoV) belongs to the family of Coronaviruses, which contains a large group of viruses that are constantly circulating in animals and humans. Illness in humans caused by coronaviruses is mostly mild and manifested by respiratory or digestive problems as leading symptoms ${ }^{1}$. However, some coronaviruses, such as SARS-CoV-1, MERS-CoV and the recent SARS$\mathrm{CoV}-2$, have been responsible for serious outbreaks of severe and lethal respiratory disease ${ }^{2,3}$. Unlike the previous outbreaks with SARS-CoV-1 and MERS-CoV, the current SARS-CoV-2 outbreak has undeniably evolved to the largest global health threat to humanity in this century.

The unprecedented scale and rapidity of the current pandemic urges the development of efficient vaccines, antiviral and anti-inflammatory drugs. A key step in expediting this process is to have animal models that recapitulate and allow to understand viral pathogenesis, and that can in particular be used to preclinically assess preventive and therapeutic countermeasures.

Acute respiratory disease caused by SARS-CoV-1 and MERS infections is characterized by a dysregulated inflammatory response in which a delayed type I interferon (IFN) response promotes the 
accumulation of inflammatory monocyte-macrophages ${ }^{4-6}$. The severe lung disease in COVID-19 patients seems to result from a similar overshooting inflammatory response. However, because even non-human primates do not fully replicate COVID-19, little information and no appropriate animal models are currently available to address this hypothesis ${ }^{7}$.

To address this knowledge gap, we compared the effect of SARS-CoV-2 infection in wild-type (WT) mice of different lineages (BALB/c and C57BL/6) and Syrian hamsters, as well as a panel of matched transgenic mouse and hamster strains with a knockout $(\mathrm{KO})$ of key components of adaptive and innate immunity. We used an original patient isolate of SARS-CoV-2 (BetaCoV/Belgium/GHB-03021/2020) that was passaged on HuH7 and Vero-E6 cells (Fig. S1 and Fig. S2A). For full characterization and to exclude possible contaminants, we performed deep sequencing on the inoculum that was used to infect the animals (Fig. S2A). No adventitious agents could be detected (data not shown). However, two in-frame deletions in the N-terminal domain and the furin-cleavage site of Spike (S) glycoprotein (9aa and 5aa, respectively) had occurred between cell culture passage $\mathrm{P} 4$ and $\mathrm{P} 6^{8-10}$, likely as adaptation to growth in Vero-E6 cells in vitro (Fig. S2B).

To first examine whether adaptive immunity contributed to the susceptibility to SARS-CoV-2 infection, we inoculated WT (immune-competent) and SCID mice (lacking functional T and B cells) from the same BALB/c background intranasally with a high $2 \times 10^{5} \mathrm{TCID}_{50}$ viral dose (P4 virus) (Fig. 1A). On day 3 p.i., a viral RNA peak in the lungs was observed (Fig. 1B and Fig. S3) with no obvious differences in viral loads (Fig. 1B) nor lung pathology (Fig. 1D and Fig. S4A and S4B) between WT and SCID mice. These data indicate that mice that lack the human ACE2 receptor ${ }^{11}$, can in principle be infected with SARS-CoV-2, although inefficiently and likely transiently, as also observed for SARS-CoV-1 ${ }^{4,12}$. However, adaptive immunity did not markedly contribute to this low susceptibility.

Interferons are prototypic first-line innate immune mechanisms on viral infections. To evaluate interferons, we compared viral RNA levels and lung pathology in WT C57BL/6 mice, and C57BL/6 mice with a genetic ablation of their type I (Ifnarl $\left.{ }^{-/}\right)$and III interferon (IFN) receptors $\left(\right.$Il2 $\left.^{-1 /}\right)$ (Fig. 1A). Ifnar $1^{-1}$ mice showed a slightly, but significantly enhanced replication of SARS-CoV-2 in the lung on day 3 p.i. compared to both WT and $I l 28 r^{-/}$mice (Fig. 1C). Similar to BALB/c mice, overall viral loads were low. Likewise, Ifnar $1^{-/}$mice that were treated with human convalescent SARS-CoV2 patient serum (HCS) prior to infection (Fig. 1E) had a one $\log _{10}$ reduction in viral loads, down to the residual input RNA levels observed in mice inoculated with inactivated SARS-CoV-2 (Fig. 1C). This provides further evidence for active, although inefficient virus replication.

WT and knockout mouse strains, all on C57BL/6 background, presented consistently with only a mild lung pathology. However, Ifnar $1^{-/}$mice showed increased levels of intra-alveolar hemorrhage, 
sometimes accompanied by some peribronchiolar inflammation (Fig. 1D and Fig. S4A and S4B). Passive transfer of HCS did not result in an obvious improvement in histopathological scores (Fig. S4C), in line with other studies about partial protection from SARS-CoV-2 infection ${ }^{13,14}$ and virusinduced inflammatory responses. Further evidence for true infection and hence viral replication is provided by transcriptomic analysis (Sharma, S. et al., in press ${ }^{15}$ ) of infected lung tissues (Fig. 1F and Fig. S5), revealing (i) an upregulation of classically enriched $(\mathrm{p}<0.001)$ antiviral effector molecules ${ }^{16}$ such as $c G A S, M x 1$, IFIH1/MDA-5, IRF3, OAS1, OAS3 and PKR/EIF2AK2 (Fig. S5, Cluster 1) and (ii) downregulation of upstream regulators STAT1, STAT3 and STING/TMEM173 (Suppl Fig. S5, Cluster 3) as shown previously ${ }^{17}$. Likewise, HCS treatment modulated, at least to some extent, the observed gene expression patterns (Fig. 1F and Fig. S5, Cluster 2) as shown by decreasing Akt1 $(\mathrm{p}=0.034)$ or increasing DDX58 (RIG-I, $\mathrm{p}=0.028)$ and $c G A S(M B 21 D 1, \mathrm{p}=0.094)$ mRNA levels. In summary, our data are in line with restriction of SARS-CoV-2 infection by the interferon system in mice, and that the inflammatory response correlates with an increased virus replication. However, due to the limited virus replication, mice were considered as a poor model to study COVID-19 pathogenesis, or to assess the efficacy of vaccines and treatments.

In contrast, Syrian hamsters have been reported to be highly susceptible to SARS-CoV- ${ }^{18}$ and SARS$\mathrm{CoV}-2^{19}$ and might thus provide a small animal model to study SARS-CoV-induced pathogenicity and the involvement of the immune response in aggravating lung disease. We compared virus replication levels and lung pathology in WT hamsters and hamsters with ablated Signal Transducer and Activator of Transcription 2 (STAT2 ${ }^{-/}$lacking type I and III IFN signaling) ${ }^{20,21}$ and IL28R expression (IL28R- $a^{-}$ ${ }^{\wedge}$ lacking IFN type III signaling) (Fig. 2A). In contrast to mice, intranasal inoculation of SARS-CoV-2 in WT hamsters resulted in high viral RNA loads (Fig. 2B and Fig. S6) and infectious titers (Fig. 2C) in the lungs. Also, a marked lung pathology [median cumulative score (MCS) 9 out of maximal score of 18; IQR=8.5-10.5 (P4 virus)] characterized by a multifocal necrotizing bronchiolitis, massive leukocyte infiltration and edema (Fig. 2D and Fig. S7A and S7B). This resembles histopathological findings in humans suffering from severe bronchopneumonia ${ }^{22}$.

For many respiratory viruses, including SARS-CoV-1, type I and III interferon signaling has been described to play an important role in restricting infection ${ }^{23}$. No marked differences were observed in viral RNA levels in the lung of WT, STAT2 ${ }^{-/}$or IL28R- $a^{-/}$hamsters (Fig. 2B). However, STAT2 $2^{-1-}$ hamsters had higher titers of infectious virus in the lung (Fig. 2C), high titer viremia ${ }^{24}$ (Fig. 2E) and high levels of viral RNA in the spleen, liver and upper and lower gastrointestinal tract ${ }^{25}$ (Fig. 2F) in comparison with WT and $I L 28 R-a^{-/-}$hamsters. Together, these data suggest STAT2 is critical for restricting SARS-CoV-2 systemic spread and suppressing viral replication outside of the lung compartment. Inversely, the observed lung pathology was much attenuated in STAT2 ${ }^{-\digamma}$ hamsters [MCS=3; IQR=1.5-3 (P4 virus)] with a limited infiltration of polymorphonuclear leukocytes 
correlated with the detection of few apoptotic bodies in the bronchus walls (Fig. 2D and Fig. S7B). On the contrary, IL28R-a $a^{-/}$hamsters showed clear signs of bronchopneumonia and peribronchiolar inflammation, yet of an intermediate score [MCS=7; IQR=6.5-7 (P4 virus)] (Fig. 2D and Fig. S7B). Matrix metalloprotease (MMP)-9 levels, which may serve as a sensitive marker for the infiltration and activation of neutrophils in inflamed tissues ${ }^{26,27}$, were markedly elevated in the lungs of all infected hamsters (Fig. 2G). However, higher MMP-9 levels were found in $S T A T 2^{-/}$animals, thereby inversely correlating with the histological findings (Fig. 2D). In addition, biomarkers elevated in critically ill COVID-19 patients ${ }^{2,28,29}$ such as the cytokines IL-6, IL-10 and IFN- $\gamma$ were not found to be markedly elevated in the serum of infected hamsters (Fig. S8B). Nonetheless, infected STAT2 ${ }^{-\digamma}$ and IL28R- $a^{-1-}$ had clearly increased levels of IL-6 and IL-10 in their lungs (Fig. S8A). Such an inverse correlation between biomarkers and pathology in WT versus $S T A T 2^{-/}$hamsters is in line with findings in mouse models of SARS-CoV-1 infection in which pathology correlated with the induction and dysregulation of alternatively activated "wound-healing" monocytes/macrophages ${ }^{4,6}$.

The lack of readily accessible serum markers or the absence of overt disease symptoms in hamsters prompted us to establish a non-invasive means to score for lung infection and SARS-CoV-2 induced lung disease by computed tomography (CT) as used in standard patient care to aid COVID-19 diagnosis with high sensitivity and monitor progression/recovery ${ }^{28-31}$. Similar as in humans ${ }^{32}$, semiquantitative lung pathology scores were obtained from high-resolution chest micro-CT scans of freebreathing animals ${ }^{33}$. Manifest consolidations were present in SARS-CoV-2 infected WT and IL28R- $a$ ${ }^{\wedge}$ hamsters, but not in $S_{T A T 2}{ }^{-/}$hamsters (Fig. 3A-B and Fig. S9A). In the upper airways, no differences were observed between WT and STAT2 ${ }^{-/}$hamsters, whereas IL28R $-\mathrm{a}^{-/-}$hamsters presented with an obvious dilation of bronchi (Fig. 3A and 3C). Further quantitative analysis ${ }^{34}$ revealed an increase of the non-aerated lung volume in SARS-CoV-2-infected WT and IL28R- $a^{-/-}$ hamsters, yet again not in STAT2 ${ }^{-/}$hamsters (Fig. 3D, Fig. S9B). Hence apart from lung consolidations and airway dilation as the main observed pathology, marked differences in other micro-CT-derived markers of specific lung pathology, such as hyperinflation, emphysema, or atelectasis $^{34,35}$ could not be observed, except in one animal that presented with hyperinflation (Fig. S9C and Fig. S10A-C). A matched comparison of the micro-CT-derived lung scores and viral loads in the lungs showed that, in line with our previous results, type I IFN responses downstream of STAT2 signaling drive lung pathology, yet has minor impact on viral replication in the lungs (Fig. 3E). Together, these data fully support micro-CT as a convenient adjunct to histological scoring (Fig. 2D and Fig. S9D) to visualize and quantify SARS-CoV-2-induced lung injury in the hamster model. Moreover, it may allow to monitor the impact of therapeutic measures non-invasively during disease progression. 


\section{Discussion}

The development of efficient therapeutic interventions against SARS-CoV-2 asks for relevant small animal models that mimic the different clinical manifestations of COVID-19 and that provide fundamental mechanistic insight in the underlying pathology/pathogenesis. Transgenic mice expressing hACE2, the bona fide receptor of SARS-CoV-1 and SARS-CoV-2 ${ }^{36}$, have been suggested as COVID-19 model. We here demonstrate that also WT mice are susceptible to SARS-CoV-2 infection, yet resulting in very limited viral replication and inflammatory responses. Ablation of type I interferon signaling in Ifnar-- mice results in the same small incremental 10-fold increase in viral replication as was reported for SARS-CoV-2 in $h A C E 2$ transgenic mice ${ }^{36,37}$. Most likely, neither mouse model will fully recapitulate pathogenesis of COVID-19, nor allow the study of clinical SARS-CoV-2 isolates.

By contrast, SARS-CoV-2 infection and associated pathology in hamsters seems to resemble what has been reported for SARS-CoV-1 in the same model. An early peak of active virus replication was noted in the lungs with viremia and extra-pulmonary spread. This was accompanied by a strong acute inflammatory response ${ }^{18}$ (as visualized by histopathology), the levels of which were correlated with those of MMP-9, a clinically relevant biomarker. Furthermore, micro-CT as established in this study may become a key instrument to non-invasively and quantitatively monitor SARS-CoV-2 lung disease. This will allow to conveniently monitor the effect of therapeutic strategies and test the preclinical efficacy of vaccine candidates.

By using unique knock-out hamster lines, we demonstrated that STAT2 plays a critical role in mediating antiviral responses and restricting systemic dissemination of SARS-CoV-2. This is in line with the effect of STATI in a mouse model of SARS-CoV-1 infection ${ }^{38}$. However and much in contrast to what is generally observed for viral infections in Stat $^{-/}$mice $^{39}$ or STAT2 $2^{-/}$hamsters ${ }^{21,40,41}$, the severe pathology induced by SARS-CoV-2 in WT hamsters is not observed in the absence of STAT2. Indeed, pneumonia as assessed by sensitive micro-CT was absent in STAT2 $2^{-/}$hamsters. Considering the negative regulation of IL- 6 and other mediators of inflammation by STAT2 ${ }^{39,42}$, our hamster model may help to understand the immune pathogenesis of Acute Lung Injury (ALI) caused by highly-pathogenic coronaviruses ${ }^{18,23,43}$ as well as other respiratory viruses ${ }^{4}$.

The increase in replication of SARS-CoV-2 seen in $I L 28 R-a^{-/}$hamsters, on one hand, combined with a tempered inflammatory response and lung injury as compared to WT hamsters, on the other hand, is in line with the role of type III IFN plays during respiratory virus infections, including SARS-CoV$1^{44}$. This observation also suggests that in humans pegylated IFN-lambda ${ }^{45,46}$ (or similar modulators of 
innate immunity) may possibly be considered to protect medical staff and other frontline workers from SARS-CoV-2 infection or to dampen symptoms in critically ill patients ${ }^{47}$.

In conclusion, hamsters may be a preferred above mice as infection model for the preclinical assessment of antiviral therapies, of convalescent serum transfer and of approaches that aim at tempering the COVID-19 immune pathogenesis in critically ill patients ${ }^{48,49}$. The latter may be achieved by repurposing anti-inflammatory drugs such as IL-6 receptor antagonists (e.g. Tocilizumab) ${ }^{50}$, or small molecule Jak/STAT inhibitors (e.g. Ruxolitinib or Tofacitinib). Educated by our finding that STAT2 signaling plays a dual role in also limiting viral dissemination, targeting the virus-induced cytokine response and overshooting of macrophage activation may need to be complemented by (directly acting) antivirals ${ }^{51}$.

\section{Methods}

\section{Animals}

Wild-type Syrian hamsters (Mesocricetus auratus) were purchased from Janvier Laboratories. All other mouse (C57BL/6, Ifnar $1^{-/}, I l 28 r^{-/}, \mathrm{BALB} / \mathrm{c}$ and SCID) and hamster $\left(S T A T 2^{-/}\right.$and IL28R- $\left.a^{-/}\right)$ strains were bred in-house. Six- to eight-weeks-old female mice and wild-type hamsters were used throughout the study. Knock-out hamsters were used upon availability; seven- to twelve-week old female $S T A T 2^{-/}$hamsters; five- to seven-week-old $I L 28 R-a^{-/-}$hamsters.

Ifnar $1^{-/}$mouse breeding couples were a generous gift of Dr. Claude Libert, IRC/VIB, University of Ghent, Belgium. Il28r $r^{-/}$mice [C57B/6N-A <tm1Brd> Ifnlr1<tm1a(EUCOMM)Wtsi>/Wtsi, strain ID: EM:07988] were provided by the Wellcome Trust Sanger Institute Mouse Genetics Project (Sanger $\mathrm{MGP}^{52}$.

$S T A T 2^{-/}$and IL28R- $a^{-/}$hamsters were generated by CRISPR/Cas-mediated gene targeting. To ablate STAT2 (Gene ID: 101830537) expression, a 1-nt frameshift mutation was introduced in exon 4 resulting in multiple premature stop codons ${ }^{20}$; to ablate IL28R (IFNLRI; Gene ID: 101833778) expression, a 22-nucleotides deletion was introduced in exon 2 resulting in multiple premature stop codons in the original open reading frame.

Animals were housed individually (hamsters) or per 5 (mice) in individually ventilated isolator cages (IsoCage N Biocontainment System, Tecniplast) with access to food and water ad libitum, and cage enrichment (cotton and cardboard play tunnels for mice, wood block for hamsters). Housing conditions and experimental procedures were approved by the ethical committee of KU Leuven (license P015-2020), following institutional guidelines approved by the Federation of European Laboratory Animal Science Associations (FELASA). Animals were euthanized by $100 \mu 1$ (mice) or $500 \mu$ l (hamsters) of intraperitoneally administered Dolethal $(200 \mathrm{mg} / \mathrm{ml}$ sodium pentobarbital, Vétoquinol SA). 
Prior to infection, the animals were anesthetized by intraperitoneal injection of a xylazine $(16 \mathrm{mg} / \mathrm{kg}$, XYL-M®, V.M.D.), ketamine (40 mg/kg, Nimatek, EuroVet) and atropine (0.2 mg/kg, Sterop) solution. Each animal was inoculated intranasally by gently adding $50 \mu 1$ droplets of virus stock containing $2 \times 10^{5} \mathrm{TCID}_{50}$ (P4 virus) or $2 \times 10^{6} \mathrm{TCID}_{50}$ (P6 virus) on both nostrils.

\section{Cells, virus and sera}

Vero-E6 (African green monkey kidney) and $\mathrm{HuH7}$ (human hepatoma) cells were maintained in minimal essential medium (Gibco) supplemented with $10 \%$ fetal bovine serum (Integro), $1 \%$ bicarbonate (Gibco), and 1\% L-glutamine (Gibco). All assays involving virus growth were performed using $2 \%$ fetal bovine serum instead of $10 \%$.

SARS-CoV-2 strain BetaCov/Belgium/GHB-03021/2020 (EPI ISL 407976|2020-02-03) recovered from a nasopharyngeal swab taken from a RT-qPCR-confirmed asymptomatic patient returning from Wuhan, China beginning of February $2020^{53}$ was directly sequenced on a MinION platform (Oxford Nanopore) as described previously ${ }^{54}$. Phylogenetic analysis confirmed a close relation with the prototypic Wuhan-Hu-1 2019-nCoV (GenBank accession number MN908947.3) strain. Infectious virus was isolated by serial passaging on HuH7 and Vero-E6 cells (see Figure S1). Virus used for animal experiments was from passages P4 and P6. Prior to inoculation of animals, virus stocks were confirmed to be free of mycoplasma (PlasmoTest, InvivoGen) and other adventitious agents by deep sequencing on a MiSeq platform (Illumina) following an established metagenomics pipeline ${ }^{55,56}$. The infectious content of virus stocks was determined by titration on Vero-E6 cells by the SpearmanKärber method. All virus-related work was conducted in the high-containment BSL3+ facilities of the KU Leuven Rega Institute (3CAPS) under licenses AMV 30112018 SBB 21920180892 and AMV 23102017 SBB 21920170589 according to institutional guidelines.

Matched human convalescent serum (HCS) was donated from the same patient under informed consent.

\section{RNA extraction and RT-qPCR}

Animals were euthanized at different time-points post-infection, organs were removed and lungs were homogenized manually using a pestle and a 12 -fold excess of cell culture medium (DMEM/2\%FCS). RNA extraction was performed from homogenate of $4 \mathrm{mg}$ of lung tissue with RNeasy Mini Kit (Qiagen), or 50 $\mu 1$ of serum using the NucleoSpin kit (Macherey-Nagel), according to the manufacturer's instructions. Other organs were collected in RNALater (Qiagen) and homogenized in a bead mill (Precellys) prior to extraction. Of $100 \mu$ l eluate, $4 \mu 1$ was used as template in RT-qPCR reactions. RT-qPCR was performed on a LightCycler96 platform (Roche) using the iTaq Universal Probes One-Step RT-qPCR kit (BioRad) with primers and probes (Table S1) specific for SARS-CoV2 , mouse $\beta$-actin and hamster $\beta$-actin (IDT). For each data point, qPCR reactions were carried out in duplicate. Standards of SARS-CoV-2 cDNA (IDT) and infectious virus were used to express the 
amount of RNA as normalized viral genome equivalent (vge) copies per mg tissue, or as TCID $_{50}$ equivalents per $\mathrm{mL}$ serum, respectively. The mean of housekeeping gene $\beta$-actin was used for normalization. The relative fold change was calculated using the $2^{-\Delta \Delta C t} \operatorname{method}^{57}$.

\section{Quantification of SARS-CoV-2 infectious particles in lung tissues}

After extensive transcardial perfusion with PBS, lungs were collected, extensively homogenized using manual disruption (Precellys24) in minimal essential medium $(5 \% \mathrm{w} / \mathrm{v})$ and centrifuged $(12,000 \mathrm{rpm}$, $10 \mathrm{~min}, 4^{\circ} \mathrm{C}$ ) to pellet the cell debris. Infectious SARS-CoV-2 particles were quantified by means of endpoint titrations on confluent Vero-E6 cell cultures. Viral titers were calculated by the SpearmanKärber method and expressed as the $50 \%$ tissue culture infectious dose $\left(\mathrm{TCID}_{50}\right)$ per $100 \mathrm{mg}$ tissue.

\section{Differential gene expression and bioinformatics analysis}

To study differential gene expression, RNA was extracted from lung tissues using Triazol, subjected to cDNA synthesis (High Capacity cDNA Reverse Transcription Kit, Thermo Fisher Scientific), and qPCR using a custom Taqman qRT-PCR array (Thermo Fisher Scientific) of 30 genes known to be activated in response to virus infection ${ }^{15}$, as well as two housekeeping genes (Table S2). Data collected were analysed using the Quant Studio Design and Analysis (version 1.5.1) and Data Assist software (version 3.01, Thermo Fischer Scientific). Pathway, GO (Gene Ontology) and transcription factor target enrichment analysis was performed using GSEA (Gene Set Enrichment Analysis, Molecular Signatures Database (MSigDB), Broad Institute). Principal component analysis, correlation matrices, unsupervised hierarchical clustering (Eucledian distance) were performed using XLSTAT and visualized using MORPHEUS (https://software.broadinstitute.org/morpheus) as described previously $^{58}$.

\section{Histology}

For histological examination, the lungs were fixed overnight in $4 \%$ formaldehyde and embedded in paraffin. Tissue sections $(4 \mu \mathrm{m})$ were stained with hematoxylin and eosin to visualize and score for lung damage.

\section{Tissue and serum biomarker analysis}

Cytokine levels in lung homogenates and serum of hamsters were determined by ELISA for IFN- $\gamma$ (EHA0005), IL-6 (EHA0008) and IL-10 (EHA0006) following the manufacturer's instructions (Wuhan Fine Biotech Co., Ltd).

The levels of gelatinase B/metalloproteinase (MMP)-9 present in lung homogenates were analyzed using gelatin zymography ${ }^{59}$, essentially as described previously ${ }^{60}$. For quantification of zymolytic bands internal control samples were spiked into each sample. Equivalent hamster enzyme 
concentrations were calculated with the use of known amounts of recombinant human pro-MMP-9 and recombinant human pro-MMP-9 $\Delta$ OGHem as standards ${ }^{61}$.

\section{Micro-computed tomography (CT) and image analysis}

Hamsters were anaesthetized using isoflurane (Iso-Vet) (2-3\% in oxygen) and installed in prone position into the X-cube micro-CT scanner (Molecubes) using a dedicated imaging bed. Respiration was monitored throughout. A scout view was acquired and the lung was selected for a non-gated, helical CT acquisition using the High-Resolution CT protocol, with the following parameters: $50 \mathrm{kVp}$, 960 exposures, $32 \mathrm{~ms} /$ projection, $350 \mu \mathrm{A}$ tube current, rotation time $120 \mathrm{~s}$. Data were reconstructed using a regularized statistical (iterative) image reconstruction algorithm using non-negative least squares $^{62}$, using an isotropic $100 \mu \mathrm{m}$ voxel size and scaled to Hounsfield Units (HUs) after calibration against a standard air/water phantom. The spatial resolution of the reconstruction was estimated at $200 \mu \mathrm{m}$ by minimizing the mean squared error between the 3D reconstruction of the densest rod in a micro-CT multiple density rod phantom (Smart Scientific) summed in the axial direction and a digital phantom consisting of a 2D disk of $17.5 \mathrm{~mm}$ radius that was post-smoothed with Gaussian kernels using different full width half maxima (FWHM), after aligning the symmetry axis of the rod to the zaxis.

Visualization and quantification of reconstructed micro-CT data was performed with DataViewer and CTan software (Bruker micro-CT). As primary outcome parameter, a semi-quantitative scoring of micro-CT data was performed as previously described ${ }^{33,34,63}$ with minor modifications towards optimization for COVID-19 lung disease in hamsters. In brief, visual observations were scored (from $0-2$ depending on severity, both for parenchymal and airway disease) on 5 different, predefined transversal tomographic sections throughout the entire lung image for both lung and airway disease by two independent observers (L.S. and G.V.V.) and averaged. Scores for the 5 sections were summed up to obtain a score from 0 to 10 reflecting severity of lung and airway abnormalities compared to scans of healthy, WT control hamsters. As secondary measures, image-derived biomarkers (nonaerated lung volume, aerated lung volume, total lung volume, the respective densities within these volumes and large airways volume) were quantified as in ${ }^{33,63}$ for a manually delineated VOI in the lung, avoiding the heart and main blood vessels. The threshold used to separate the airways and aerated (0-55) from non-aerated lung volume (56-255) was set manually on a 8-bit greyscale histogram and kept constant for all data sets.

\section{Statistical analysis}

GraphPad Prism (GraphPad Software, Inc.) was used for all statistical evaluations. The number of animals and independent experiments that were performed is indicated in the legends to figures. Statistical significance was determined using the non-parametric Mann Whitney U-test. Values were considered significantly different at $\mathrm{P}$ values of $\leq 0.05$. 


\section{Acknowledgments}

We thank Kathleen Van den Eynde, Eef Allegaert, Sarah Cumps, Wilfried Versin, Caroline Collard, Elke Maas and Jasper Rymenants for excellent technical assistance. We thank Johan Nuyts (Nuclear Medicine and Molecular Imaging, KU Leuven) for support with imaging file processing, Pieter Mollet (Molecubes, Ghent, Belgium) for outstanding technical support with the micro-CT installation, Laurens Liesenborghs (Molecular Vaccinology and Vaccine Discovery, KU Leuven) for help with graphics, Jef Arnout and Annelies Sterckx (KU Leuven Faculty of Medicine, Biomedical Sciences Group Management) and Animalia and Biosafety Departments of KU Leuven for facilitating the studies. We also thank Dr. Claude Libert (IRC/VIB, University of Ghent) for providing the Ifnar -/mice and the Wellcome Trust Sanger Institute Mouse Genetics Project (Sanger MGP) and its funders for providing the $I l 28 r$-/- mutant mouse line.

This project has received funding from the European Union's Horizon 2020 research and innovation program under grant agreements No 101003627 (SCORE project) and No 733176 (RABYD-VAX consortium), and was supported by the Research Foundation Flanders (FWO) under the Excellence of Science (EOS) program (VirEOS project 30981113), the FWO Hercules Foundation (Caps-It infrastructure), the KU Leuven Rega Foundation, and the Stichting Antoine Faes. J.M. was supported by a grant from the China Scholarship Council (CSC). C.C. was supported by the FWO (FWO 1001719N). G.V.V. acknowledges grant support from KU Leuven Internal Funds (C24/17/061) and K.D. grant support from KU Leuven Internal Funds (C3/19/057 Lab of Excellence). G.O. and E.M. were supported by C16/17/010 funding from KU Leuven and FWO-Vlaanderen.

\section{Author Contributions}

R.B., H.J.T. J.N. and K.D. designed experiments;

R.B., S.J.F.K, R.L., V.V., C.D.K., S.S., E.M., L.B., T.V.B., J.M. and W.C. carried out experiments; R.B., H.J.T., L.S., S.J., J.V.W., E.M., B.W., C.C., G.V.V., Z.W. and K.D. analyzed data; L.D., J.R.P., J.M., G.S., K.V.L., G.O. and P.M. provided advice on the interpretation of data;

R.B., H.J.T., and K.D wrote the original draft with input from co-authors;

R.B., H.J.T., L.S., J.V.W., C.C., G.V.V., J.N. and K.D. wrote the final draft;

R.L., Y.L., Z.W., and M.V.R. provided essential reagents;

H.E., D.S., and P.L. provided and facilitated access to essential infrastructure;

H.J.T., J.N. and K.D. supervised the study;

K.D., L.C., P.L. and J.N. acquired funding;

All authors approved the final manuscript.

\section{Declaration of Interests}

The authors declare no competing interests. 
A

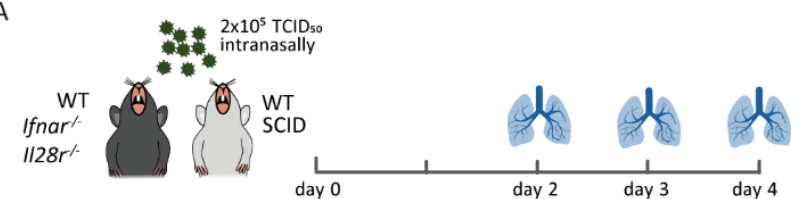

B Viral RNA in lung

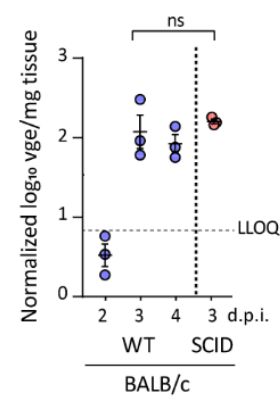

D Lung pathology

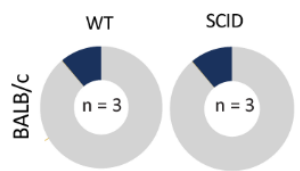

Peribronchiolar inflammation
C Viral RNA in lung
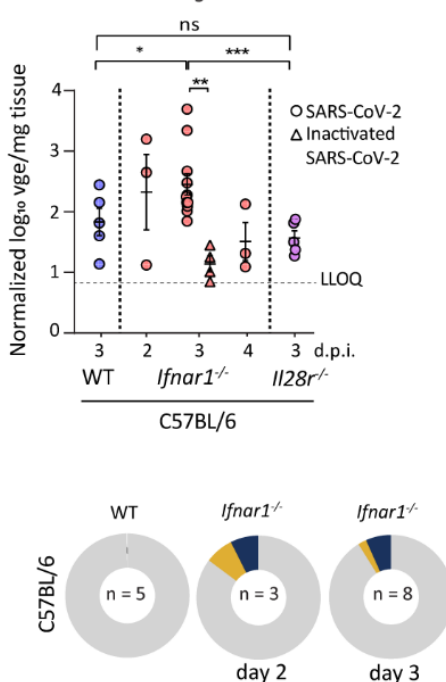

E Viral RNA in lung in Ifnar ${ }^{\%}$
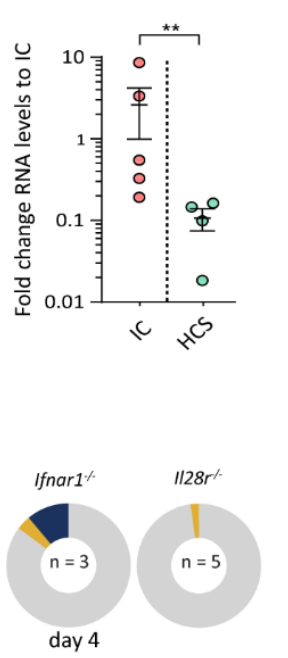

day 4
F Gene expression heatmap Ifnar1\%

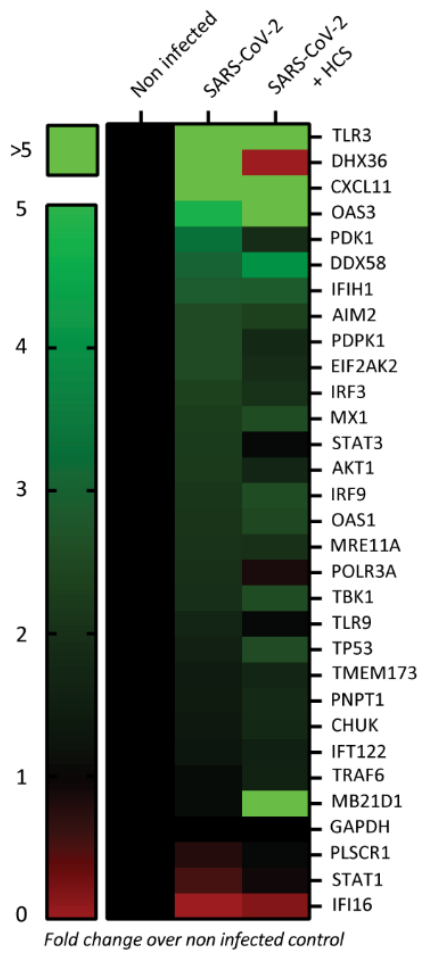

Figure 1. Type I interferon signaling restricts infection of the lungs of mice. (A) Schematic representation of SARS-CoV-2 inoculation schedule. Several wild-type (WT) and knock-out mouse strains were intranasally inoculated with $2 \times 10^{5}$ TCID $_{50}$ of passage 4 (P4) SARS-CoV2. Convalescent serum treatment was given intraperitoneally (i.p.) 1 day prior to inoculation. On the indicated days post inoculation (d.p.i.), lungs were collected for determination of viral RNA levels and scored for lung damage. (B-C) Normalized viral RNA levels in the lungs of BALB/c WT and SCID mice and C57BL/6 WT, Ifnarl ${ }^{-/}$and $I l 28 r^{-/}$mice. At the indicated time intervals p.i., viral RNA levels were determined by RT-qPCR, normalized against $\beta$-actin mRNA levels and transformed to estimate viral genome equivalents (vge) content per weight of the lungs (Figure S2). For heat-inactivation, SARS-CoV-2 was incubated for $30 \mathrm{~min}$ at $56^{\circ} \mathrm{C}$. Dotted line indicates lower limit of quantification (LLOQ). (D) Histopathological scoring of lungs for all different mouse strains. Mice were sacrificed on day 3 p.i. and lungs were stained with $\mathrm{H} \& \mathrm{E}$ and scored for signs of lung damage (inflammation and hemorrhage). Scores are calculated as percentage of the total maximal score. "No score" means not contributing to theoretical full cumulative score of $100 \%$. Numbers (n) of animals analyzed per condition are given in the inner circle (E) Viral RNA levels in Ifnar $^{-/-}$mice after treatment with anti-SARSCoV2 sera. Mice were either left untreated (IC, infection control) or treated with HCS (human convalescent serum) and sacrificed on day 3 p.i. Viral RNA levels were determined in the lungs, normalized against $\beta$-actin and fold-changes were calculated using the $2^{(-\Delta \Delta \mathrm{Cq})}$ method compared to mean of IC. The data shown are means \pm SEM. (F) Heatmap showing gene expression profiles of 30 selected marker genes in the lungs of uninfected $(n=3)$ and infected Ifnar $^{-/}$mice that were either left untreated $(n=5)$ or treated with $\operatorname{HCS}(n=4)$. The scale represents fold change compared to non-infected animals. Statistical significance between groups was calculated by the nonparametric Mann-Whitney U-test (ns $\mathrm{P}>0.05$, * $\mathrm{P}<0.05$, ** $\mathrm{P}<0.01, * * * \mathrm{P}<0.001)$. 
A

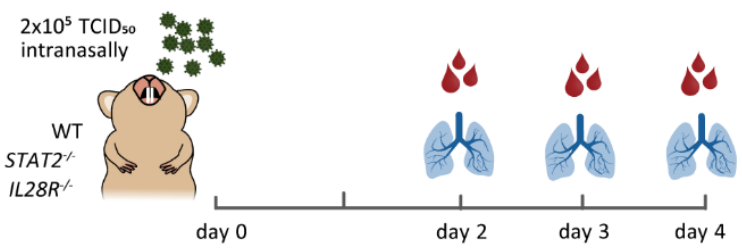

D Lung pathology

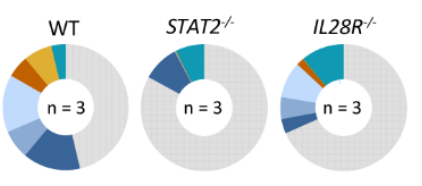

E Viral RNA in blood

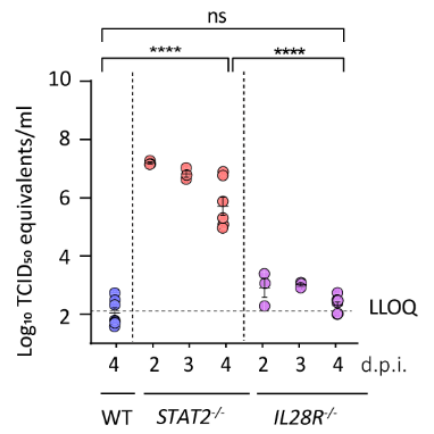

B Viral RNA in lung

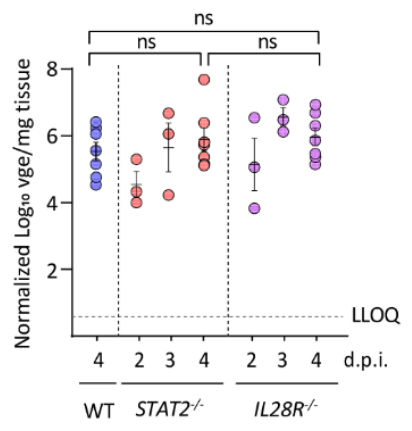

C Infectious virus in lung

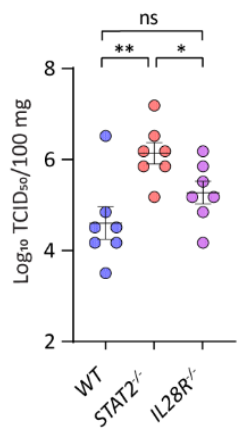

Figure 2. Exuberant innate response by STAT2 drives SARS-CoV-2-induced lung pathology in hamsters. (A) Schematic representation of SARS-CoV-2 inoculation schedule. WT, STAT2 ${ }^{-/}$and IL28R- $a^{-/}$hamster strains were intranasally inoculated with $2 \times 10^{5}$ TCID $_{50}$ of passage 4 or $2 \times 10^{6}$ of passage 6 SARS-CoV-2. On the indicated days post inoculation (d.p.i.), organs and blood were collected to determine viral RNA levels, infectious virus load and score for lung damage. Viral loads in the indicated organs were quantified by RT-qPCR $(\mathbf{B}, \mathbf{E}$ and $\mathbf{F})$ or virus titration $(\mathbf{C})$. (B,F) Viral RNA levels in the indicated organs were normalized against $\beta$-actin mRNA levels and transformed to estimate viral genome equivalents (vge) content per weight of the lungs (Figure S5). (C) Infectious virus loads in the lung are expressed as the number of infectious virus particles per $100 \mathrm{mg}$ of lung tissue. (E) Viral RNA levels in the blood were calculated from a standard of infectious virus and expressed as TCID 50 equivalents per ml blood. Dotted lines indicate lower limit of quantification (LLOQ) or lower limit of detection (LLOD) (D) Histopathological scoring of lungs. Hamsters were sacrificed on day 4 p.i. with passage 4 SARS-CoV-2 and lungs were stained with H\&E and scored for signs of lung damage (apoptotic bodies, necrotizing bronchiolitis, edema, pneumonia and inflammation). Scores are calculated as percentage of the total maximal score. (G) Levels of matrix metalloproteinase (MMP)-9 levels in lung homogenates of SARS-CoV-2 infected hamsters, relative to non-infected controls of the same strain. Values for infected animals $(n=7$ each) compiled from two independent experiments using either P4 $(n=3$, circles) and P6 $(n=4$, squares) SARS-CoV-2. Statistical significance was calculated between infected and noninfected animals within each group. The data shown are mean \pm SEM. Statistical significance between groups was calculated by the nonparametric Mann-Whitney U-test (ns P > 0.05, * P $<0.05, * * \mathrm{P}<0.01, * * * * \mathrm{P}<0.0001)$. 
A $\mu$-CT images of infected lungs

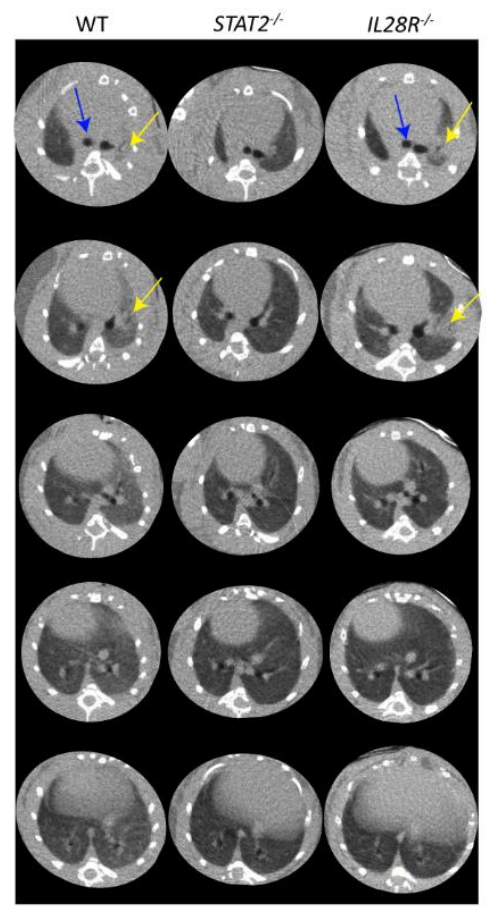

B $\mu$-CT-derived lung score

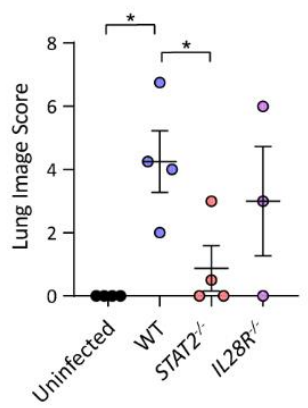

E Matched comparison $\mu$-CT score and infectious titers in the lung

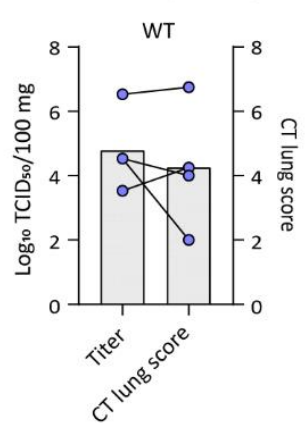

C $\mu$-CT-derived bronchi score
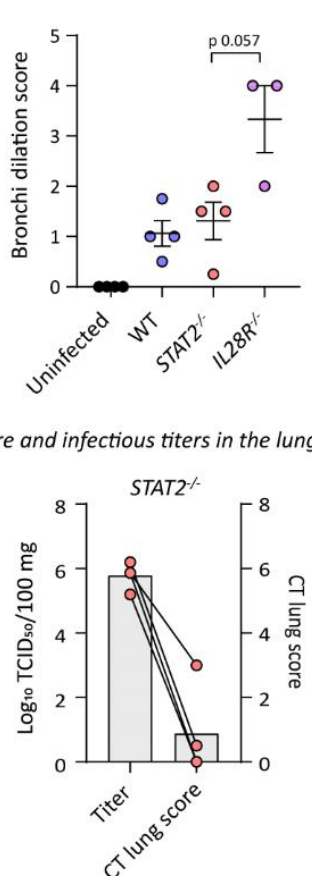

D $\mu$-CT-derived volume
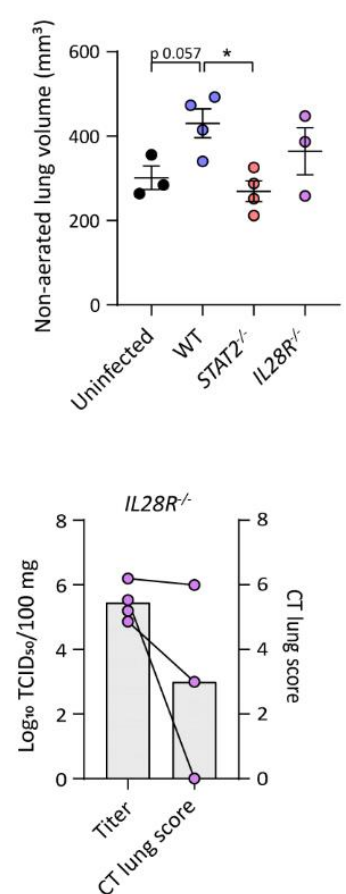

Figure 3. Micro-CT reveals severe lung injury in hamsters. (A) Representative transversal micro-CT images of infected (P6 SARS-CoV-2) WT, STAT2 ${ }^{-/}$and $I L 28 R-a^{-/-}$hamster lungs at 4 d.p.i. ( $n=4$ each). Arrows indicate examples of pulmonary infiltrates seen as consolidation of lung parenchyma (yellow) or dilatation of upper airways (blue). Five transverse cross sections at different positions in the lung were selected for each animal and scored to quantify lung consolidations (B) or dilatation of the bronchi (C). (D) Quantification of the micro-CT-derived non-aerated lung volume biomarker, reflecting the volume consolidations in the lungs. (E) Matched comparison between micro-CT-derived lung scores (B) and infectious virus load in the lung (Fig. 2C). Lines indicate matched samples. The data shown are mean \pm SEM. Statistical significance between groups was calculated by the nonparametric Mann Whitney Utest (ns $\mathrm{P}>0.05, * \mathrm{P}<0.05)$. 


\section{References}

1. Cui, J., Li, F. \& Shi, Z. L. Origin and evolution of pathogenic coronaviruses. Nature Reviews Microbiology vol. 17 181-192 (2019).

2. Zhu, N. et al. A novel coronavirus from patients with pneumonia in China, 2019. N. Engl. J. Med. 382, 727-733 (2020).

3. Chen, J. et al. Clinical progression of patients with COVID-19 in Shanghai, China. J. Infect. 80, e1 (2020).

4. Channappanavar, R. et al. Dysregulated Type I Interferon and Inflammatory MonocyteMacrophage Responses Cause Lethal Pneumonia in SARS-CoV-Infected Mice. Cell Host Microbe 19, 181-193 (2016).

5. Channappanavar, R. \& Perlman, S. Pathogenic human coronavirus infections: causes and consequences of cytokine storm and immunopathology. Seminars in Immunopathology vol. 39 529-539 (2017).

6. Page, C. et al. Induction of Alternatively Activated Macrophages Enhances Pathogenesis during Severe Acute Respiratory Syndrome Coronavirus Infection. J. Virol. 86, 13334-13349 (2012).

7. Rockx, B. et al. Comparative pathogenesis of COVID-19, MERS, and SARS in a nonhuman primate model. Science (80-. ). eabb7314 (2020) doi:10.1126/science.abb7314.

8. Andersen, K. G., Rambaut, A., Lipkin, W. I., Holmes, E. C. \& Garry, R. F. The proximal origin of SARS-CoV-2. Nat. Med. 26, 450-452 (2020).

9. Zhang, T., Wu, Q. \& Zhang, Z. Probable Pangolin Origin of SARS-CoV-2 Associated with the COVID-19 Outbreak. Curr. Biol. 30, 1346-1351.e2 (2020).

10. Wrapp, D. et al. Cryo-EM structure of the 2019-nCoV spike in the prefusion conformation. Science (80-. ). 367, 1260-1263 (2020).

11. Hoffmann, M. et al. SARS-CoV-2 Cell Entry Depends on ACE2 and TMPRSS2 and Is Blocked by a Clinically Proven Protease Inhibitor. Cell 181, 271-280.e8 (2020).

12. Ishii, K. et al. Neutralizing antibody against severe acute respiratory syndrome (SARS)coronavirus spike is highly effective for the protection of mice in the murine SARS model. Microbiol. Immunol. 53, 75-82 (2009).

13. Shen, C. et al. Treatment of 5 Critically Ill Patients with COVID-19 with Convalescent Plasma. JAMA - J. Am. Med. Assoc. (2020) doi:10.1001/jama.2020.4783.

14. Gralinski, L. E. et al. Complement activation contributes to severe acute respiratory syndrome coronavirus pathogenesis. MBio 9, (2018).

15. Sharma, S. et al. (in press) Small molecule inhibitors of TBK1 serve as adjuvant for a plasmidlaunched live-attenuated yellow fever vaccine. Hum. Vaccines Immunother. (2020).

16. Schoggins, J. W. Interferon-Stimulated Genes: What Do They All Do? Annu. Rev. Virol. 6, 567-584 (2019).

17. Khouri, R. et al. A genetic IFN/STAT1/FAS axis determines CD4 T stem cell memory levels and apoptosis in healthy controls and Adult T-cell Leukemia patients. Oncoimmunology 7 , (2018).

18. Roberts, A. et al. Severe acute respiratory syndrome coronavirus infection of golden Syrian hamsters. J. Virol. 79, 503-11 (2005).

19. Chan, J. F.-W. et al. Simulation of the clinical and pathological manifestations of Coronavirus Disease 2019 (COVID-19) in golden Syrian hamster model: implications for disease pathogenesis and transmissibility. Clin. Infect. Dis. (2020) doi:10.1093/cid/ciaa325.

20. Fan, Z. et al. Efficient gene targeting in golden Syrian hamsters by the CRISPR/Cas9 system. PLoS One 9, (2014).

21. Toth, K. et al. STAT2 Knockout Syrian Hamsters Support Enhanced Replication and Pathogenicity of Human Adenovirus, Revealing an Important Role of Type I Interferon Response in Viral Control. PLoS Pathog. 11, e1005084 (2015).

22. $\mathrm{Xu}, \mathrm{Z}$. et al. Pathological findings of COVID-19 associated with acute respiratory distress syndrome. Lancet Respir. Med. 8, 420-422 (2020).

23. Mahlakõiv, T. et al. Combined action of type I and type III interferon restricts initial replication of severe acute respiratory syndrome coronavirus in the lung but fails to inhibit 
systemic virus spread. J. Gen. Virol. 93, 2601-2605 (2012).

24. Chen, W. et al. Detectable 2019-nCoV viral RNA in blood is a strong indicator for the further clinical severity. Emerging Microbes and Infections vol. 9 469-473 (2020).

25. Lin, L. et al. Gastrointestinal symptoms of 95 cases with SARS-CoV-2 infection. Gut gutjnl2020-321013 (2020) doi:10.1136/gutjnl-2020-321013.

26. Potey, P. M. D., Rossi, A. G., Lucas, C. D. \& Dorward, D. A. Neutrophils in the initiation and resolution of acute pulmonary inflammation: understanding biological function and therapeutic potential. Journal of Pathology vol. 247 672-685 (2019).

27. Opdenakker, G., Van Den Steen, P. E. \& Van Damme, J. Gelatinase B: A tuner and amplifier of immune functions. Trends in Immunology vol. 22 571-579 (2001).

28. Wang, D. et al. Clinical Characteristics of 138 Hospitalized Patients with 2019 Novel Coronavirus-Infected Pneumonia in Wuhan, China. JAMA - J. Am. Med. Assoc. 323, 10611069 (2020).

29. Huang, C. et al. Clinical features of patients infected with 2019 novel coronavirus in Wuhan, China. Lancet 395, 497-506 (2020).

30. Ai, T. et al. Correlation of Chest CT and RT-PCR Testing in Coronavirus Disease 2019 (COVID-19) in China: A Report of 1014 Cases. Radiology 200642 (2020) doi:10.1148/radiol.2020200642.

31. Chen, T. et al. Clinical characteristics of 113 deceased patients with coronavirus disease 2019: retrospective study. BMJ 368, m1091 (2020).

32. Shi, H. et al. Radiological findings from 81 patients with COVID-19 pneumonia in Wuhan, China: a descriptive study. Lancet Infect. Dis. 20, 425-434 (2020).

33. Poelmans, J. et al. Longitudinal, in vivo assessment of invasive pulmonary aspergillosis in mice by computed tomography and magnetic resonance imaging. Lab. Investig. 96, 692-704 (2016).

34. Vande Velde, G. et al. Longitudinal micro-CT provides biomarkers of lung disease that can be used to assess the effect of therapy in preclinical mouse models, and reveal compensatory changes in lung volume. DMM Dis. Model. Mech. 9, 91-98 (2016).

35. de Langhe, E. et al. Quantification of lung fibrosis and emphysema in mice using automated micro-computed tomography. PLoS One 7, (2012).

36. Mccray, P. B. et al. Lethal Infection of K18-hACE2 Mice Infected with Severe Acute Respiratory Syndrome Coronavirus. J. Virol. 81, 813-821 (2007).

37. Yang, X. H. et al. Mice transgenic for human angiotensin-converting enzyme 2 provide a model for SARS coronavirus infection. Comp. Med. 57, 450-459 (2007).

38. Frieman, M. B. et al. SARS-CoV pathogenesis is regulated by a STAT1 dependent but a type I, II and III interferon receptor independent mechanism. PLoS Pathog. 6, 1-14 (2010).

39. Park, C., Li, S., Cha, E. \& Schindler, C. Immune response in Stat2 knockout mice. Immunity 13, 795-804 (2000).

40. Gowen, B. B. et al. Modeling Severe Fever with Thrombocytopenia Syndrome Virus Infection in Golden Syrian Hamsters: Importance of STAT2 in Preventing Disease and Effective Treatment with Favipiravir. J. Virol. 91, (2017).

41. Siddharthan, V. et al. Zika virus infection of adult and fetal STAT2 knock-out hamsters. Virology 507, 89-95 (2017).

42. Gough, D. J., Messina, N. L., Clarke, C. J. P., Johnstone, R. W. \& Levy, D. E. Constitutive Type I Interferon Modulates Homeostatic Balance through Tonic Signaling. Immunity vol. 36 166-174 (2012).

43. Liu, L. et al. Anti-spike IgG causes severe acute lung injury by skewing macrophage responses during acute SARS-CoV infection. JCI insight 4, (2019).

44. Mordstein, M. et al. Lambda interferon renders epithelial cells of the respiratory and gastrointestinal tracts resistant to viral infections. J. Virol. 84, 5670-7 (2010).

45. Yurdaydin, C. New treatment options for delta virus: Is a cure in sight? Journal of Viral Hepatitis vol. 26 618-626 (2019).

46. Andreakos, E., Zanoni, I. \& Galani, I. E. Lambda interferons come to light: dual function cytokines mediating antiviral immunity and damage control. Current Opinion in Immunology vol. 56 67-75 (2019). 
47. Prokunina-Olsson, L. et al. COVID-19 and emerging viral infections: The case for interferon lambda. J. Exp. Med. 217, (2020).

48. Zumla, A., Hui, D. S., Azhar, E. I., Memish, Z. A. \& Maeurer, M. Reducing mortality from 2019-nCoV: host-directed therapies should be an option. The Lancet vol. 395 e35-e36 (2020).

49. Liu, Q., Zhou, Y. H. \& Yang, Z. Q. The cytokine storm of severe influenza and development of immunomodulatory therapy. Cellular and Molecular Immunology vol. 13 3-10 (2016).

50. Kang, S., Tanaka, T., Narazaki, M. \& Kishimoto, T. Targeting Interleukin-6 Signaling in Clinic. Immunity vol. 50 1007-1023 (2019).

51. Agostini, M. L. et al. Coronavirus susceptibility to the antiviral remdesivir (GS-5734) is mediated by the viral polymerase and the proofreading exoribonuclease. MBio $\mathbf{9}$, (2018).

52. White, J. K. et al. XGenome-wide generation and systematic phenotyping of knockout mice reveals new roles for many genes. Cell 154, 452 (2013).

53. Spiteri, G. et al. First cases of coronavirus disease 2019 (COVID-19) in the WHO European Region, 24 January to 21 February 2020. Eurosurveillance vol. 25 (2020).

54. Vrancken, B. et al. Accounting for population structure reveals ambiguity in the Zaire Ebolavirus reservoir dynamics. PLoS Negl. Trop. Dis. 14, e0008117 (2020).

55. Conceição-Neto, N. et al. Modular approach to customise sample preparation procedures for viral metagenomics: A reproducible protocol for virome analysis. Sci. Rep. 5, 16532-16532 (2015).

56. Conceição-Neto, N., Yinda, K. C., Van Ranst, M. \& Matthijnssens, J. NetoVIR: Modular approach to customize sample preparation procedures for viral metagenomics. in Methods in Molecular Biology vol. 1838 85-95 (Humana Press Inc., 2018).

57. Livak, K. J. \& Schmittgen, T. D. Analysis of relative gene expression data using real-time quantitative PCR and the 2- $\Delta \Delta$ CT method. Methods 25, 402-408 (2001).

58. Van Weyenbergh, J., Wietzerbin, J., Rouillard, D., Barral-Netto, M. \& Liblau, R. Treatment of multiple sclerosis patients with interferon-beta primes monocyte-derived macrophages for apoptotic cell death. J. Leukoc. Biol. 70, 745-8 (2001).

59. Vandooren, J., Geurts, N., Martens, E., Van Den Steen, P. E. \& Opdenakker, G. Zymography methods for visualizing hydrolytic enzymes. Nature Methods vol. 10 211-220 (2013).

60. Descamps, F. J., Martens, E. \& Opdenakker, G. Analysis of Gelatinases in Complex Biological Fluids and Tissue Extracts. Lab. Investig. 82, 1607-1608 (2002).

61. Felix, K. et al. Differential Diagnosis of Autoimmune Pancreatitis from Pancreatic Cancer by Analysis of Serum Gelatinase Levels. Pancreas 45, 1048-1055 (2016).

62. Vandeghinste, B. et al. Iterative ct reconstruction using shearlet-based regularization. IEEE Trans. Nucl. Sci. 60, 3305-3317 (2013).

63. Berghen, N. et al. Radiosafe micro-computed tomography for longitudinal evaluation of murine disease models. Sci. Rep. 9, (2019). 